\title{
Early Stages of Secondary Phase Formation in Multicomponent Alloys Using an in situ TEM Heating Approach
}

Elaf A. Anber ${ }^{1}$, Eric A. Lass ${ }^{2}$, Andrew C Lang ${ }^{1}$, Pranav Kumar Suri ${ }^{1}$, Daniel Scotto D’Antuono ${ }^{1}$, Haoyan Diao ${ }^{3}$, Rui Feng ${ }^{3}$, Peter K Liaw ${ }^{3}$ and Mitra L Taheri ${ }^{1}$

${ }^{1 .}$ Department of Materials Science and Engineering, Drexel University, Philadelphia, PA, USA.

2. Materials Science and Engineering Division, National Institute of Standards and Technology, Gaithersburg, MD, USA.

3. Department of Materials Science and Engineering, University of Tennessee Knoxville, Knoxville, TN, USA.

The equimolar CoCrFeNi (with minor addition of Al) high entropy alloys (HEAs), has been widely studied recently due to their unique properties including high thermal stability and high toughness at the cryogenic temperature. The main concepts behind establishing high entropy alloys was to promote formation of stable solid solutions, such as FCC and BCC structures due to the effect of high configurational entropy of these alloys [1-2]. However, despite the unique core effects of these alloys, the growth of secondary phases during thermal ageing at intermediate and high annealing temperature has been frequently reported, attracting a particular attention concerning the alloy design in terms of structural properties and potential applications. While the formation of these phases enhances the overall mechanical properties [3], it may negatively impact the formation of productive oxide layers and thus deteriorates the corrosion properties when the alloy exposed to a corrosive environment such as $\mathrm{NaCl}[4,5]$.

In this study, we employ the in-situ TEM heating technique to track the early, intermediate and final stages of second phase precipitation in $\mathrm{Al}_{\mathrm{x}} \mathrm{CoCrFeNi}(\mathrm{x}=0.0,0.1,0.3,0.5)$ HEA. As-homogenized HEAs were insitu heat treated in a JEOL $2100 \mathrm{LaB}_{6} \mathrm{TEM}$, equipped with a high-resolution pole piece, using a Gatan heating holder at ramp rate of $0.5 \%$ s. Scanning transmission electron microscope (STEM)-equipped with energy dispersive x-ray spectroscopy (EDS) was used to study the chemical composition of the precipitates. Following STEM-EDS, selected area diffraction patterns (SAED) technique was performed to examine the crystal structure of as-homogenized and annealed samples. We find that NiAl, Co-rich and Cr-rich precipitates formed in this alloy after undergoing in-situ TEM heating at $550{ }^{\circ} \mathrm{C}, 700{ }^{\circ} \mathrm{C}$, and 900 ${ }^{\circ} \mathrm{C}$ for different annealing periods. We found that by increasing the Al addition, the precipitates density increase, where the formation of $\mathrm{B} 2$ phases being more dominant.

The study was coupled with phase and orientation analysis using precession electron diffraction techniques to examine the effect of grain boundary character on the precipitation of second phases.

Figure 1(a-f) shows the representative high-angle annular dark-field (HAADF)-(STEM), and EDS analysis of in-situ TEM annealed $\mathrm{Al}_{0.3} \mathrm{CoCrFeNi} \mathrm{HEAs}$ at $700^{\circ} \mathrm{C}$ for $1 \mathrm{hr}$.

Activation energy calculations will be performed to obtain quantitative data from the in-situ heating experiments, where the study aimed to understand the effect of chemical composition (addition of Al) on activation energy. This work required acquiring high quality in-situ heating video of single precipitates and measuring the values of time, temperature and area, allowing the usage of the Arrhenius equation [6]:

$$
k=\operatorname{Ae}\left(\begin{array}{c}
Q \\
R T
\end{array}\right)
$$

where K: the rate constant, A: frequency factor, Q: activation energy, R: gas constant and T: temperature in kelvin. 
Overall, the work described provides a foundation for predictive understanding of phase evolution, opening the window toward designing innovative alloys for targeted applications. These results are discussed in the context of the growing literature comparing the ideal methods for stabilizing mechanisms in HEAs for use in high temperature environments.

\section{References:}

[1] Li et al., Acta Mater. 123 (2017), p. 285.

[2] U Dahlborg et al., J. Alloys Compd. 681 (2016), p. 330.

[3] Shun et al., J. Alloys Compd. 479 (2009), p. 157.

[4] Miracle et al., Acta Mater. 122 (2017), p. 448.

[5] Shi et al., Appl. Surf. Sci. 439 (2018), p. 533.

[6] D'Antuono et al., Acta Materialia 123 (2017), p. 264.
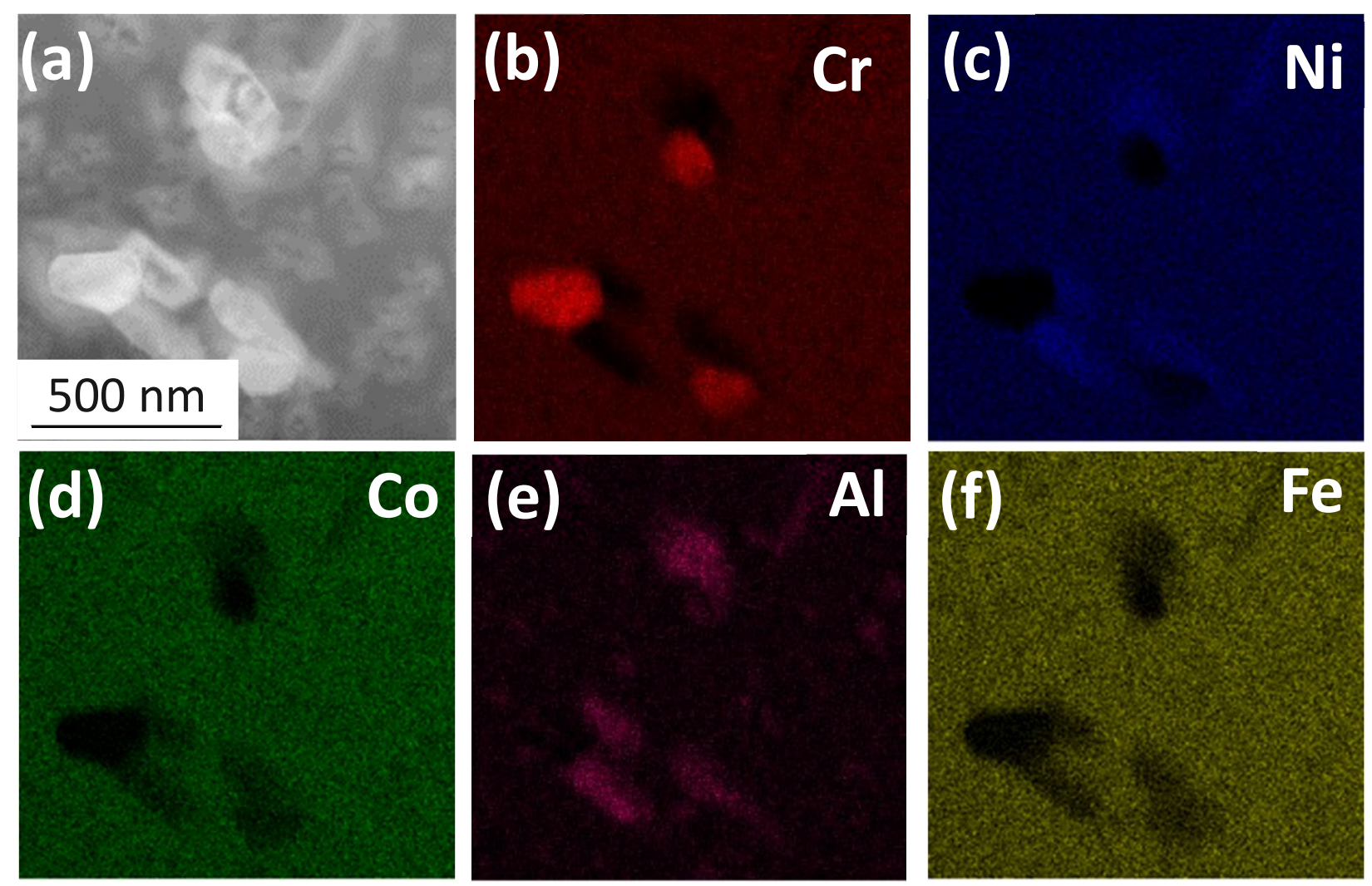

Figure 1. Representative HADAAF-STEM image of $\mathrm{Al}_{0.3} \mathrm{CoCrFeNi}(\mathrm{a})$, EDS elemental maps show the distribution of $\mathrm{Cr}(\mathrm{b}), \mathrm{Ni}(\mathrm{c}), \mathrm{Co}(\mathrm{d}), \mathrm{Al}(\mathrm{e})$ and $\mathrm{Fe}(\mathrm{f})$. 